\title{
Fluorescence imaging of lattice re-distribution on step-index direct laser written Nd:YAG waveguide lasers
}

\author{
Jon Martínez de Mendívil, ${ }^{1}$ Airán Ródenas, ${ }^{2,3}$ Antonio Benayas, ${ }^{1,4, a)}$ Magdalena Aguiló, ${ }^{2}$ \\ Francesc Diaz, ${ }^{2}$ Alberto Pérez Delgado, ${ }^{1}$ Ginés Lifante, ${ }^{1}$ Daniel Jaque, ${ }^{1}$ and Ajoy K. Kar ${ }^{3}$ \\ ${ }^{1}$ Departamento de Física de Materiales, Facultad de Ciencias, Universidad Autónoma de Madrid, \\ Madrid 28049, Spain \\ ${ }^{2}$ Departament de Química Física i Inorgànica, Universitat Rovira i Virgili, Tarragona 43007, Spain \\ ${ }^{3}$ Institute of Photonics and Quantum Sciences, Heriot-Watt University, Edinburgh EH14 4AS, United Kingdom \\ ${ }_{4}^{4}$ Institut National de la Recherche Scientifique, Centre - Énergie Matériaux et Télécommunications; 1650, \\ Boul. Lionel Boulet Varennes, Quebec J3X 1S2, Canada
}

(Received 24 September 2014; accepted 19 December 2014; published online 12 January 2015)

\begin{abstract}
The laser performance and crystalline micro-structural properties of near-infrared step-index channel waveguides fabricated inside Neodymium doped YAG laser ceramics by means of three-dimensional sub-picosecond pulse laser direct writing are reported. Fluorescence micro-mapping of the waveguide cross-sections reveals that an essential crystal lattice re-distribution has been induced after short pulse irradiation. Such lattice re-distribution is evidenced at the waveguide core corresponding to the laser written refractive index increased volume. The waveguides core surroundings also present diverse changes including slight lattice disorder and bi-axial strain fields. The step-index waveguide laser performance is compared with previous laser fabricated waveguides with a stress-optic guiding mechanism in absence of laser induced lattice re-distribution. (C) 2015 AIP Publishing LLC. [http://dx.doi.org/10.1063/1.4905318]
\end{abstract}

\section{INTRODUCTION}

The volume inscription of localized refractive index modifications is at the basis of the success of the ThreeDimensional (3D) Direct Laser Writing (DLW) technique. ${ }^{1-3}$ DLW has been extensively used for the fabrication of $3 \mathrm{D}$ buried waveguides in a great variety of transparent materials including glasses, crystals, and transparent ceramics. ${ }^{4}$ DLW has been found especially appropriate for the local modification and controlled fabrication of laser waveguides in rare earth doped YAG crystals and ceramics. ${ }^{5-12}$ In the simplest channel waveguide fabrication scheme (in which waveguides are constituted by either a single or double laser-written line tracks), waveguiding is produced at the stress affected volume (generally called Type II stress-induced waveguides) where the refractive index changes are produced through the piezo-optic effect. ${ }^{10}$ In other cases, waveguiding is produced by a low-index cladding. ${ }^{13,14}$ Both types of structures are typically fabricated using low-repetition rate lasers $(1 \mathrm{kHz})$ and have been successfully used for the demonstration of efficient Nd:YAG waveguides lasers operating at $1.06 \mu \mathrm{m}$ $\left({ }^{4} \mathrm{~F}_{3 / 2} \rightarrow{ }^{4} \mathrm{I}_{11 / 2}\right.$ transition of Neodymium ions). ${ }^{7,8,15}$ One of the main drawbacks of Nd:YAG DLW waveguides fabricated with low repetition rate lasers is the unavoidable damage and stress, as well as the impossibility of writing increased step-index type waveguides. The importance of being able to inscribe step-index increased waveguides (Type I WGs in the following), relies on the fact that these type of waveguides (step-index direct written cores) could exhibit superior light confinement and lower bend losses, as


antonio.benayas@emt.inrs.ca
}

well as much more controllable mode dispersion properties, than stress-induced waveguides. ${ }^{16}$ Moreover, they allow for the direct inscription of controllable size cores, with controllable core index increments, which make it fundamental for the design of 3D optical circuits, especially in combination with microfluidics.

Recently, it was demonstrated that step-index profile waveguides can be also fabricated in Nd:YAG by using medium pulse repetition rates $(100 \mathrm{kHz})$ and the so-called multi-scan (MS) DLW technique. ${ }^{17}$ MS-DLW uses subpicosecond pulses with energies in the order of hundreds of nano-Joules and medium pulse repetition rates $(100 \mathrm{kHz}$ to $1 \mathrm{MHz}$ ) leading to localized positive refractive index changes of up to $\sim 0.01$ within the focal volume. ${ }^{18}$ MS-DLW of waveguides has been demonstrated in different crystals such as $\mathrm{LiNbO}_{3}, \mathrm{ZnSe}, \mathrm{YCOB}$ and recently in YAG. ${ }^{11,12,17-19}$ The use of larger repetition rates is probably the main enabling factor to refractive index increment since it leads to the appearance of controlled heat accumulation and self-annealing effects which are absent at lower repetition rates. ${ }^{20,21}$ Due to the possibility of precisely achieving the desired core size and having refractive index changes similar to standard NIR fibers, these type of waveguides can exhibit very low coupling losses to fibers of around $0.1 \mathrm{~dB}$, this being an important feature for fiber-coupled photonic chips. Recent works have indeed demonstrated that YAG WGs fabricated by MS-DLW were capable of light guiding at infrared wavelengths $(1.9 \mu \mathrm{m}) .{ }^{17}$ Despite their capability for efficient infrared light confinement, MS-DLW waveguides have not been yet fabricated in Neodymium doped YAG transparent laser ceramics. The resulting structures would be of high technological interest as integrated infrared laser sources. In addition, the hypersensitive fluorescence 
lines of Neodymium ions can be used as unique tools to elucidate the microstructural modifications induced by the MS technique and, thus, to answer fundamental questions regarding the microstructural changes leading to the refractive index modifications.

In this work, we have fabricated buried channel waveguides in Nd:YAG ceramics by the MS-DLW technique and lased them for the first time to our knowledge. The ability of the fabricated structures to confine IR light has been demonstrated and, in addition, potential application as integrated continuous wave laser structures has been evaluated. The micro-structural modifications induced during the MS-DLW procedure have been elucidated from the high resolution confocal fluorescence images of waveguides obtained in terms of the hyper-sensitive fluorescence lines of Neodymium ions.

\section{RESULTS AND DISCUSSION}

Waveguides were fabricated in a $2 \%$ Neodymium doped YAG ceramic sample (provided by Baikowsky Ltd) with an average gran size of $2 \mu \mathrm{m}$. MS-DLW was performed by using an IMRA $\mu$ Jewel mode-locked laser system $(100 \mathrm{kHz}$ repetition rate, $1047 \mathrm{~nm}$ central wavelength). The laser beam was focused $100 \mu \mathrm{m}$ below the sample's surface by using an aspheric lens (0.6 NA). Pulse duration was studied from $350 \mathrm{fs}$ to $650 \mathrm{fs}$, and the generation of refractive-index increased step-index waveguides was found to appear only for $650 \mathrm{fs} .{ }^{17}$ Scans were performed in a zig-zag pattern, the separation between consecutive parallel scans was set to $0.6 \mu \mathrm{m}$, and the scan speed was set to $0.1 \mathrm{~mm} / \mathrm{s}$. Pulse energies were tested between 250 and $290 \mathrm{~nJ}$. The waveguides show a long-term stability, without any sign of deterioration for more than 3 years since fabrication. Fig. 1(b) shows an optical transmission image of the waveguide fabricated by using $280 \mathrm{~nJ}$ pulses, this being an optimum writing energy in terms of insertion losses. The square WG cross section can be seen. The capability of the fabricated WG to guide nearinfrared light was verified by end-coupling a $1.45 \mu \mathrm{m}$ fiber coupled diode laser. The near field image of the propagating mode at the exit face of the Nd:YAG WG was measured by a cooled InGaAs infrared camera. Results are included in Fig. 1(c). As can be observed, the $1.45 \mu \mathrm{m}$ propagation mode
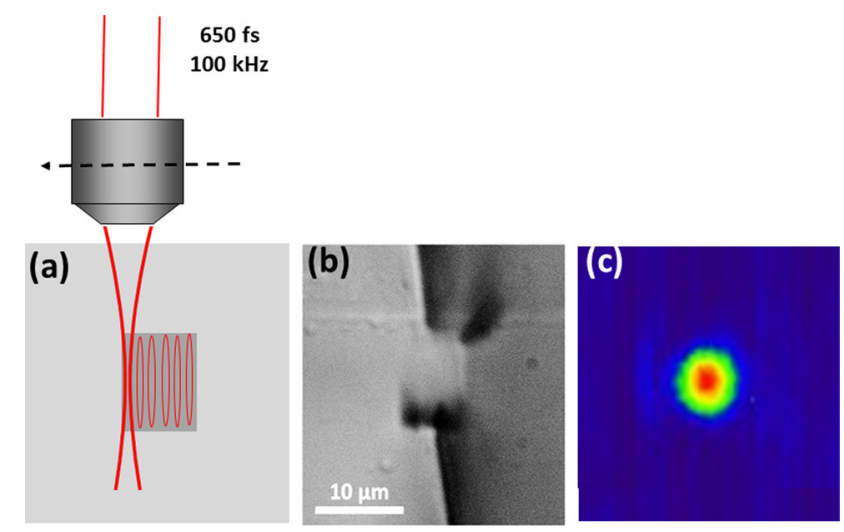

FIG. 1. (a) Scheme of the laser writing process. (b) Optical transmission image of step-index waveguide. (c) Near field image of the $1.45 \mu \mathrm{m}$ output mode. was found to be highly symmetric; this being a desirable feature for optical interconnects with other devices such as single mode fibers.

The luminescence properties of the MS-DLW WG were also analyzed by using a confocal micro-luminescence mapping setup. A $808 \mathrm{~nm}$ laser beam provided by a single-mode fiber coupled diode laser was focused into the microstructured Nd:YAG sample by using a $0.85 \mathrm{NA}$ long working distance microscope objective. In this work, we focus our study on the spectral variations induced in the Neodymium fluorescence line at around $940 \mathrm{~nm}$ that correspond to the ${ }^{4} \mathrm{~F}_{3 / 2}\left(\mathrm{R}_{2}\right) \rightarrow{ }^{4} \mathrm{I}_{9 / 2}\left(\mathrm{Z}_{5}\right)$ Stark transition. The intensity, bandwidth, and peak position of this particular fluorescence line have been extensively used as precise spectroscopic indicators of the local damage, disorder, and distortion of the YAG lattice, respectively. ${ }^{10,20}$

Figure 2 shows this fluorescence line as obtained at the waveguide core (B) and at an unprocessed (A) area. The spectrum obtained from an unprocessed area agrees well with previous works as it is constituted by a narrow and symmetric band centered at around $938.9 \mathrm{~nm} .{ }^{10}$ On the contrary, the emission spectrum obtained from the WG core volume is completely different. It is constituted by the superposition of two fluorescence lines centered at around 939 and $940 \mathrm{~nm}$, with an energy separation between them close to $11 \mathrm{~cm}^{-1}$. This is in contrast with the case of Nd:YAG stress-field based Type II WGs, in which the fluorescence generated at WG volume was composed by a single line. ${ }^{10}$

According to Lupei et al. multi-line emission in $\mathrm{Nd}$ :YAG ceramics denotes a multi-site distribution of Neodymium ions in the YAG lattice, i.e., the presence of Neodymium ions in different non-equivalent sites within the YAG lattice. ${ }^{22,23}$ When all the $\mathrm{Nd}^{3+}$ ions replace Yttrium ions in the YAG lattice (single site distribution) ${ }^{4} \mathrm{~F}_{3 / 2} \rightarrow{ }^{4} \mathrm{I}_{9 / 2}$ Stark transitions leads to single line fluorescence bands. ${ }^{22}$ On the other hand, if a lattice re-distribution is produced, Neodymium ions occupy different lattice sites so that additional fluorescence lines could be observed due to single Stark transitions with different emission energies and, very



FIG. 2. Fluorescence emission spectra of the line centered at $938.9 \mathrm{~nm}\left({ }^{4} \mathrm{~F}_{3 / 2}\right.$ $\left(\mathrm{R}_{2}\right) \rightarrow{ }^{4} \mathrm{I}_{9 / 2}\left(\mathrm{Z}_{5}\right)$ transition of $\mathrm{Nd}^{3+}$ ions) as obtained from an unprocessed area (A), and in the waveguide core (B). Dashed blue and green lines show the spectral deconvolution of emission lines performed in this work. 
likely, different radiative and nonradiative probabilities. ${ }^{24,25}$ Independently on the exact origin of new emission lines, what it is evident is that the "distorted" emission spectrum obtained in the irradiated volume unequivocally indicates an essential lattice re-distribution among the YAG lattice that has been produced at the at the laser focal volume. The ultrafast laser induced modification of ionic species is not a new phenomenon as it has been previously reported in glasses in which laser induced ion diffusion has been reported to occur in several systems. ${ }^{26}$ Nevertheless, experimental data included in Figure 2 constitutes to our knowledge the first evidence of the possibility of using sub-picosecond laser pulses to promote the permanent and localized lattice re-distribution in a crystalline environment (thus modifying rare earth ions' fluorescence emission shape). At this point, it should be noted that in previous studies the use of $350 \mathrm{fs}$ pulse durations at $100 \mathrm{kHz}$ did not produce the refractiveindex increment observed for $650 \mathrm{fs}$ pulses. This is very likely due to an insufficient thermal energy deposition, as it is possible with temporally longer pulses of $650 \mathrm{fs}$ width. There are different explanations to account for the larger thermal loading produced when using longer pulses. First, pulse lengths below $1 \mathrm{ps}$ are expected to produce a reduced heat affected zone than pulses longer that $1 \mathrm{ps}$, for this reason the term ultra-short pulse interaction is generally used for pulses shorter than a picosecond. The characteristic time required for energy exchange from electrons to the lattice (ions) system is in the order of ps (what in previous papers has been denoted as electron-phonon coupling). The description of an electron-phonon system as a two-fluid system with different temperatures and thermal diffusion times is commonly used to describe laser-plasma interactions. ${ }^{27-29}$ Secondly, the temperature within the focal region does also depend on the fluence (energy per unit area), and a common effect in femtosecond laser writing in optical media is the appearance of self-focusing effects as a result of too high pulse peak powers (energy per unit time of a pulse). In our case, the use of $340 \mathrm{fs}$ pulses and around $300 \mathrm{~nJ}$ pulse energies implies that we are working on the threshold for selffocusing in YAG and filamentation occurs, as we showed in our previous work-see Fig. 1(b) within Rodenas et al. ${ }^{17}$ However, using a double length pulse (660 fs), the pulse power is well below the threshold for self-focusing and the pulse energy is fully deposited within a more confined volume, thus potentially increasing the local temperature at the focal volume.

Figures 3(a) and 3(b) show, respectively, the spatial variation, over the WG cross section, of the peak position and spectral width of the normal site emission line $\left(\mathrm{Nd}^{3+}\right.$ ions occupying the Yttrium sites in the YAG lattice). These fluorescence images can be unequivocally correlated with the stress and disorder defect distributions. The main peak has been red-shifted at both laterals of the WG that evidences an axial lattice compression in these zones as it is characteristic from previous studies. ${ }^{10}$ Such a lattice compression is accompanied by a slight lattice dilatation due to tensile stress at the top and bottom of the WG, where the main fluorescence line has been blue-shifted. At the WG core (indicated by the dashed line in Fig. 3) no appreciable spectral shift in
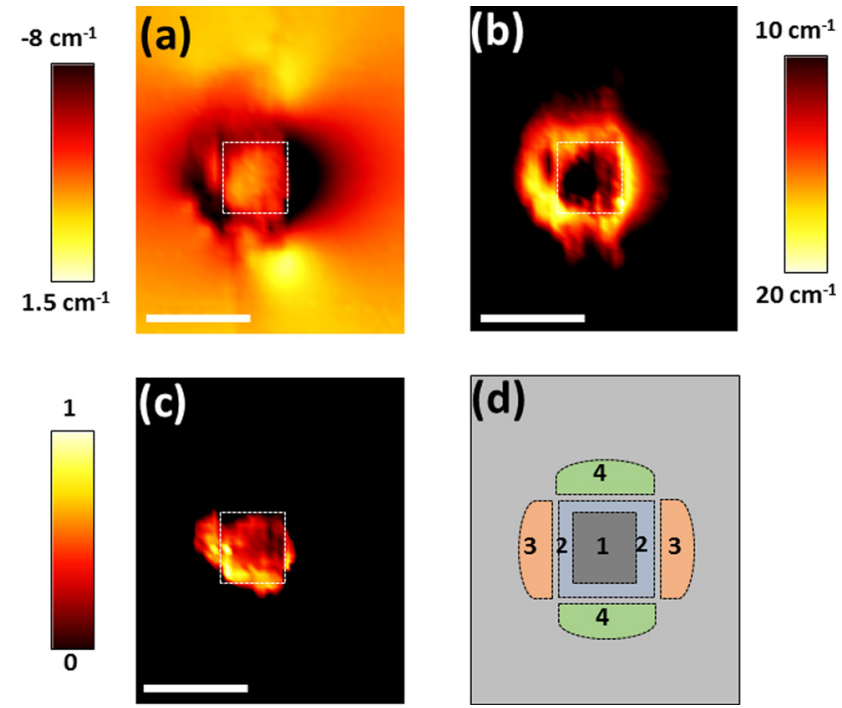

FIG. 3. Confocal microscopy images of the waveguide cross section as obtained for the spectral shift (a) and width (b) of the fluorescence peak at $938.9 \mathrm{~nm}$ and associated to the $\mathrm{Nd}^{3+}$ ions at Yttrium site. (c) Emission intensity of the new $\mathrm{Nd}^{3+}$-emission line at around $940 \mathrm{~nm}$ appearing as a consequence of the laser-induced lattice re-distribution. The white dashed-line square indicates the position of the core. Scale bar is $10 \mu \mathrm{m}$ in all cases. (d) Scheme of the four different regions: (1) $\mathrm{Nd}^{3+}$ re-distributed ions area, (2) strongly disordered YAG lattice, (3) axially compressed volume, (4) tensile stressed volumes.

the fundamental line has been observed. This indicates that, in the irradiated volume, the crystal field affecting $\mathrm{Nd}^{3+}$ ions at normal Yttrium sites does not differ from that of unprocessed material, suggesting the absence of residual stress (average inter-atomic distances in the YAG lattice are the same as those of non-processed areas). The linewidth map image (see Fig. 3(c)) agrees with these observations as it denotes the presence of a disordered (modified) YAG lattice only at the surroundings of the WG core, where residual stress has been localized. Fig. 3(c) shows the integrated area mapping of the additional line attributed to $\mathrm{Nd}^{3+}$ ions affected by the lattice re-distribution. As can be observed, this additional line only contributes significantly to the overall fluorescence at the WG core volume. This means that the severe lattice re-distribution has been only induced at the irradiated volume. Based on the fluorescence images of Figs. 3(a)-3(c), we propose the schematic structural image shown in Fig. 3(d). The WG active core volume is constituted by a severe re-distribution of the YAG network has been induced as a consequence of both fs laser irradiation and thermal accumulation processes (region 1 in Fig. 3(d)). This region is surrounded by a strongly disordered YAG volume in which $\mathrm{Nd}^{3+}$ ions are retained at their original Yttrium site within the YAG lattice (region 2 in Fig. 3(d)). Finally, two lateral volumes of densified Nd:YAG (regions 3 in Fig. 3(d)) are also visible. Such densified volumes are mechanically accompanied by locally dilated volumes at the upper and bottom of the WG structure (regions 4 in Fig. 3(d)).

According to the structural image proposed in Fig. 3, the active volume of the MS-DLW WGs is constituted by an inhomogeneous line broadened material as its luminescence is generated by non-equivalent Neodymium ions. This fact 
could be of crucial importance in the laser performance of the fabricated waveguides. Laser materials with inhomogeneous line broadened emission lines are known to show reduced laser slope efficiencies, especially when output couplers with large transmittances are used. ${ }^{30}$ The possible adverse effects that lattice re-distribution could have on the laser performance of MS-DLW WGs have been investigated by performing mirror-less laser action experiments. Optical excitation was performed by using a 808 tuned Ti:Sapphire laser beam that was coupled into the MS-DLW WG by using a 0.35 NA microscope objective. Optical feed-back was achieved by the Fresnel reflections at both input and output WG facets. The $1.06 \mu \mathrm{m}$ generated laser radiation was collected by a 0.40 NA microscope objective.

Figure 4 shows the mirror-less $1.06 \mu \mathrm{m}$ laser power obtained as a function of the $808 \mathrm{~nm}$ launched power. Stable laser operation was obtained with a laser threshold and slope efficiency of $60 \mathrm{~mW}$ and $13 \%$, respectively. For the sake of comparison, Figure 4 also includes the mirror-less laser curve as obtained, in exactly the same experimental conditions, from a stress based Type II waveguides. These waveguides were constructed by using the double line approach and by using $100 \mathrm{fs}$ pulses at a repetition rate of $1 \mathrm{kHz}$ and pulse energy of $11 \mu \mathrm{J}$. More details about these waveguides can be found elsewhere. ${ }^{7}$ As commented before, these waveguides did not show any lattice re-distribution so they behave as homogeneous line broadened laser sources. In this case, the laser threshold was also close to $60 \mathrm{~mW}$, i.e., similar to that of MS-DLW WGs. On the other hand, the Type II WGs showed a much larger laser slope efficiency (close to 55\%), several times larger than that obtained from MS-DLW WGs. Although several facts could account for this marked difference in the laser slope efficiency (such as waveguide mode size, propagation losses and so on), we state at this point that the deterioration in the laser performance found for MS-DLW WGs is very likely caused by the inhomogeneous line broadening of fluorescence lines caused by the crystal lattice re-distribution (that structural change making Neodymium ions occupy different lattice sites, thus creating

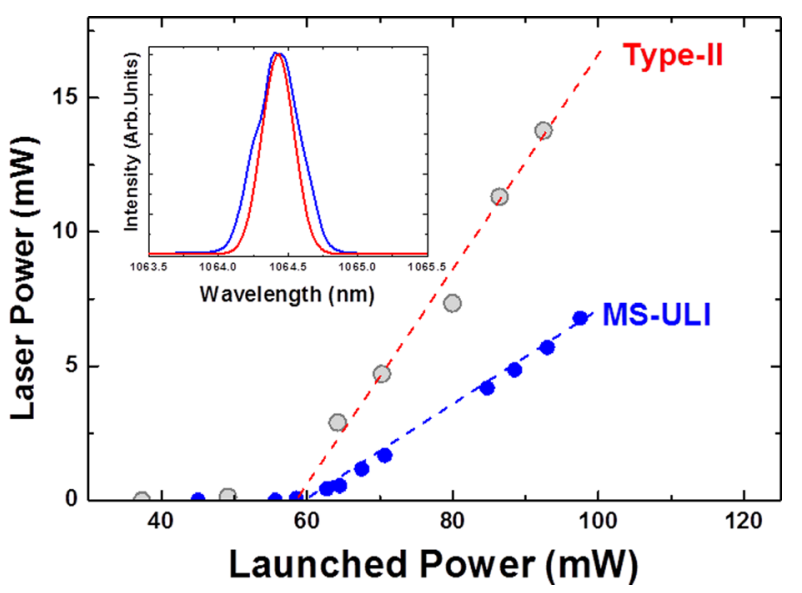

FIG. 4. Laser power at $1.06 \mu \mathrm{m}$ as a function of the launched power $(808 \mathrm{~nm})$ measured at the output of two mirrorless laser waveguides fabricated by two different techniques: Type II (red circles) and MS-DLW (blue circles). Inset shows the laser emission spectra obtained in each case. as a direct consequence the spectral broadening of the fluorescence emission from the $\mathrm{Nd}^{3+}$ ions). Note that in inhomogeneous line broadening laser systems large reduction in the laser slope efficiency are expected, especially when high transmittance couplers are used (as they are in our case were no mirrors were employed). In an inhomogeneous line broadened laser system the gain spectrum (in a first order approximation proportional to the fluorescence spectrum) is broader than for a homogeneous line broadened system. This is due to the presence of non-equivalent sites/centers for the optically active ions (Neodymium ions in our case). When the system is under laser operation and the cavity losses are low, then all the non-equivalent centers (even those with low gain) can contribute to the laser gain and in this case the laser spectrum is expected to be a "broad" laser spectrum. On the other hand, when cavity losses are high (as they are in our case due to the use of a mirror-less cavity) only those centers with the highest gain will contribute to the laser beam. Typically, only optical centers in the "non-perturbed" position will contribute to laser gain (i.e., Neodymium ions redistributed in the YAG network due to laser irradiation) do not contribute to laser oscillation. ${ }^{30}$ In our case, the laser spectrum is a narrow one (see inset in Figure 4). Indeed, it is almost as narrow as that obtained from the homogeneous line broadened Type II waveguides, as only the "original" or "non- perturbed" centers are contributing to the laser beam. In this situation, pump radiation excites all the active ions (Neodymium ions) but only a fraction of them are contributing to the laser beam. As a consequence, although the laser emission spectra could be as narrow as that of a homogeneous line broadened system, the laser slope efficiency is strongly reduced due to the presence of non-equivalent centers. Regarding mode size, it can be expected that Type-I step-index WGs will allow for superior mode confinement and therefore improved slope efficiencies in the near future. Regarding optical propagation losses, their reduction is expected to improve both lasing thresholds and efficiencies.

\section{CONCLUSIONS}

In summary, our optical investigations carried out on MS-DLW Nd:YAG waveguides have shown, for the first time, the existence of a severe lattice re-distribution as a consequence of ultrafast laser irradiation. We postulate that such lattice's ions re-distribution has been favored by heat accumulation effects inherent to the use of pulse trains with high repetition rates $(100 \mathrm{kHz})$. Finally, we state that the aforementioned crystal lattice re-distribution implies an inhomogeneous line broadening of Nd:YAG transition that leads to a deterioration in the waveguide laser performance when compared with Type II homogeneously line broadened $\mathrm{Nd}$ :YAG laser waveguides.

\section{ACKNOWLEDGMENTS}

This work was supported by the Spanish Government under Project Nos. MAT2013-47395-C4-1-R, TEC201021574-C02-01/02, MAT2013-47395-C4, and MAT201129255-C02-02 and by the Generalitat de Catalunya under Project No. 2014SGR1358. A. Benayas thanks Fonds de 
recherche du Québec-Nature et technologies (FRQNT) for the Postdoctoral Fellowship given to him through Programme de Bourses d'Excellence (Merit Scholarship Program for Foreign Students).

${ }^{1}$ E. Gamaly, B. Luther-Davies, and A. Rode, in 3D Laser Microfabrication (Wiley-VCH Verlag GmbH \& Co. KGaA, 2006), p. 5.

${ }^{2}$ S. S. Mao, F. Quéré, S. Guizard, X. Mao, R. E. Russo, G. Petite, and P. Martin, Appl. Phys. A 79, 1695 (2004).

${ }^{3}$ F. Chen and J. R. V. de Aldana, Laser Photon. Rev. 8, 251 (2014).

${ }^{4}$ G. Della Valle, R. Osellame, and P. Laporta, J. Opt. Pure Applied Opt. 11, 013001 (2009).

${ }^{5}$ A. Rodenas, A. Benayas, and A. Jaque, in Femtosecond Laser Assisted Micro-Modifications in Nd:YAG Ceramics for Photonic Applications (Tenerife, 2007).

${ }^{6}$ A. G. Okhrimchuk, A. V. Shestakov, I. Khrushchev, and J. Mitchell, Opt. Lett. 30, 2248 (2005).

${ }^{7}$ G. A. Torchia, A. Rodenas, A. Benayas, E. Cantelar, L. Roso, and D. Jaque, Appl. Phys. Lett. 92, 111103 (2008).

${ }^{8}$ H. Liu, Y. Jia, J. R. Vázquez de Aldana, D. Jaque, and F. Chen, Opt. Express 20, 18620 (2012).

${ }^{9}$ M. Lenzner, J. Krüger, S. Sartania, Z. Cheng, C. Spielmann, G. Mourou, W. Kautek, and F. Krausz, Phys. Rev. Lett. 80, 4076 (1998).

${ }^{10}$ A. Rodenas, G. A. Torchia, G. Lifante, E. Cantelar, J. Lamela, F. Jaque, L. Roso, and D. Jaque, Appl. Phys. B-Lasers Opt. 95, 85 (2009).

${ }^{11} \mathrm{~A}$. Okhrimchuk, in Coherence and Ultrashort Pulse Laser Emission, edited by F. J. Duarte (InTech, 2010), p. 519.

${ }^{12}$ A. Okhrimchuk, M. Dubov, V. Mezentsev, and I. Bennion, in Permanent Refractive Index Change Caused by Femtosecond Laser Beam in YAG Crystals (Paris, 2008), p. 15.

${ }^{13}$ H. Zhang, S. M. Eaton, and P. R. Herman, Opt. Express 14, 4826 (2006).
${ }^{14}$ A. Okhrimchuk, V. Mezentsev, A. Shestakov, and I. Bennion, Opt. Express 20, 3832 (2012).

${ }^{15}$ Y. Tan, Q. Luan, F. Liu, F. Chen, and J. R. Vázquez de Aldana, Opt. Express 21, 18963 (2013).

${ }^{16}$ A. W. Snyder and J. Love, Optical Waveguide Theory (Springer, 1983), Vol. 190.

${ }^{17}$ A. Rodenas, A. Benayas, J. R. Macdonald, J. Zhang, D. Y. Tang, D. Jaque, and A. K. Kar, Opt. Lett. 36, 3395 (2011).

${ }^{18}$ A. Rodenas and A. K. Kar, Opt. Express 19, 17820 (2011).

${ }^{19}$ J. R. Macdonald, R. R. Thomson, S. J. Beecher, N. D. Psaila, H. T. Bookey, and A. K. Kar, Opt. Lett. 35, 4036 (2010).

${ }^{20}$ A. Benayas, W. F. Silva, A. Rodenas, C. Jacinto, J. V. de Aldana, F. Chen, Y. Tan, R. R. Thomsom, N. D. Psaila, D. T. Reid, G. A. Torchia, A. K. Kar, and D. Jaque, Appl. Phys. A 104, 301 (2011).

${ }^{21}$ A. H. Nejadmalayeri and P. R. Herman, Opt. Express 15, 10842 (2007).

${ }^{22}$ V. Lupei, Opt. Mater. 19, 95 (2002).

${ }^{23}$ V. Lupei, A. Lupei, S. Georgescu, B. Diaconescu, T. Taira, Y. Sato, S. Kurimura, and A. Ikesue, J. Opt. Soc. Am. B 19, 360 (2002).

${ }^{24}$ V. V. Osiko, Y. K. Voron'ko, and A. A. Sobol, in Growth and Defect Structures, edited by H. C. Freyhardt and G. Müller (Springer, Berlin/ Heidelberg, 1984), Vol. 10, p. 37.

${ }^{25}$ M. K. Ashurov, Y. Voronko, V. V. Osiko, A. A. Sobol, and M. I. Timoshechkin, Physica Status Solidi A 42, 101 (1977).

${ }^{26}$ F. Luo, G. Lin, H. Pan, Q. Chen, J. Qiu, Q. Zhao, and Z. Xu, J. NonCrystall. Solids 357, 2384 (2011).

${ }^{27}$ W. Kruer, The Physics Of Laser Plasma Interactions (Frontiers in Physics) (Westview Press, 2003).

${ }^{28}$ M. I. Kaganov, I. M. Lifshitz, and L. V. Tanatarov, Zh. Eksp. Teor. Fiz. 31, 232 (1957) [Sov. Phys. JETP 39, 375 (1974)].

${ }^{29}$ I. U. A. e. Il'inski $\breve{1}$ and L. V. Keldysh, Electromagnetic Response of Material Media (Springer, 1994).

${ }^{30}$ N. Mermilliod, R. Romero, I. Chartier, C. Garapon, and R. Moncorge, IEEE J. Quantum Electron. 28, 1179 (1992). 